\title{
Retrospective Medical Record Review to Describe Use of Repository Corticotropin Injection Among Patients with Uveitis in the United States
}

\author{
Winnie W. Nelson, ${ }^{1}$ Antonio Flavio Lima, John Kranyak, ${ }^{1}$ Barima Opong-Owusu, Gosia Ciepielewska, \\ Jack R. Gallagher, ${ }^{2}$ Kylee Heap, ${ }^{2}$ and Susan Carroll ${ }^{2}$
}

\begin{abstract}
Introduction: Repository corticotropin injection (RCI) has immune-modulatory and anti-inflammatory effects and is approved for multiple indications, including severe and acute chronic allergic and inflammatory processes involving the eye and adnexa. This study describes patient characteristics, treatment patterns, and physicians' assessments of patients with uveitis treated with RCI.

Methods: This was a retrospective medical record review of US patients. Eligible patients had a diagnosis of uveitis, received RCI in the past 12 months, and had completed or were receiving RCI treatment at the time of data collection. Baseline characteristics and after-treatment clinical data are descriptively reported.

Results: The study included 91 patients (mean age 41 years, 62\% female, and mean time since diagnosis 3.98 years). Most patients had moderate $(n=48,53 \%)$ to severe $(n=21,23 \%)$ visual impairment, and none was blind before RCI therapy. Patients used an average of 2.5 medications before RCI. Initial RCI dosing regimens, dose adjustments, and treatment durations were different for each patient. Concomitant medication use and dosages were reduced during RCI; 76 patients (84\%) improved, 15 patients (16\%) stayed the same, and none worsened; $86 \%$ of patients had improvements in vision.

Conclusions: Physicians individualized RCI therapy among patients who suffered uveitis for several years and when previous therapies were inadequate. Most patients improved after initiating RCI, most commonly in vision. The findings support use of RCI for uveitis and provide a better understanding of patient characteristics and practice patterns to guide appropriate use.
\end{abstract}

Keywords: ACTH, drug therapy, health care utilization, repository corticotropin injection, RCI, uveitis

\section{Introduction}

$\mathbf{U}$ VEITIS IS AN inflammatory eye disease that affects both the uveal tract and adjacent structures, including the sclera, cornea, vitreous humor, retina, and optic nerve head. ${ }^{1,2}$ Symptoms of uveitis usually vary based on the site of inflammation, but decreased vision, eye pain, tearing, redness, light sensitivity, and floaters are common. ${ }^{2,3}$ Infectious and noninfectious etiologies of uveitis are known, and different treatment strategies are used based on the underlying cause. Noninfectious uveitis may be idiopathic, but is often associated with another systemic autoimmune or inflammatory diseases such as ankylosing spondylitis, sarcoidosis, juvenile idiopathic arthritis, or Behcet's disease. ${ }^{3,4}$ In the United States, the annual incidence of uveitis ranges between 24.9 and 52.4 per 100,000 persons. $^{5-7}$ The highest reported incidence comes from a Northern California population and is $\sim 3$ times higher than a historical report. ${ }^{6,8}$ The reported differences in incidence rates may reflect geographical variation, improvements in detection, or increased incidence over time.

Uveitis is associated with substantial morbidity and is a leading cause of preventable blindness in adults. ${ }^{1,3,9}$ Patients with noninfectious uveitis are at increased risk of ocular

\footnotetext{
${ }^{1}$ Mallinckrodt Pharmaceuticals, Bedminster, New Jersey.

${ }^{2}$ Clarity Pharma Research, LLC, Spartanburg, South Carolina.
}

(C) Winnie W. Nelson et al. 2019; Published by Mary Ann Liebert, Inc. This Open Access article is distributed under the terms of the Creative Commons Attribution Noncommercial License (http://creativecommons.org/licenses/by-nc/4.0/) which permits any noncommercial use, distribution, and reproduction in any medium, provided the original author(s) and the source are cited. 
complications, including cataract, glaucoma, visual disturbance, retinal detachment, and retinal disorder. ${ }^{10,11}$ Vision loss is most commonly caused by cystoid macular edema or cataract, but can be related to secondary glaucoma, vitreous opacities, retinal vascular occlusions, chorioretinal scarring, inflammatory optic neuropathy, or retinal detachment.

Treatments for noninfectious uveitis target inflammatory processes. Corticosteroids are the mainstay of treatment and are administered as topical, systemic, and intraocular therapies. Repository corticotropin injection (RCI; H.P. Acthar $\mathrm{Gel}^{\circledR}$; Mallinckrodt ARD, Hazelwood, MO) is indicated for the treatment of severe acute and chronic allergic and inflammatory processes involving the eye and its adnexa, such as keratitis, iritis, iridocyclitis, diffuse posterior uveitis and choroiditis, optic neuritis, chorioretinitis, and anterior segment inflammation. ${ }^{12} \mathrm{RCI}$ is a highly purified sterile formulation of adrenocorticotrophic hormone in $16 \%$ gelatin to provide sustained release after intramuscular or subcutaneous injection. Recommended intramuscular injection sites are upper arms or upper-outer thighs, and subcutaneous injection sites are upper arms, abdomen, or thighs. RCI stimulates the adrenal cortex to secrete cortisol, corticosterone, aldosterone, and a number of weakly androgenic substances. In addition, RCI is reported to bind to melanocortin receptors. The mechanism of action of RCI is believed to be mediated through the stimulation of low level of cortisol release and melanocortin receptor binding.

In 2010, the US Food and Drug Administration determined that sufficient evidence existed to support the indications for RCI. Nevertheless, the literature on RCI use in patients with uveitis remained limited. To date, there are 2 published case reports, both indicating favorable response to treatment. ${ }^{13,14}$ Additional data are needed to guide appropriate use of this medication in the treatment of uveitis.

The purpose of this study was to gather data from a large case series of patients with uveitis who were treated with RCI. The objectives were to describe patient demographic and clinical characteristics, utilization patterns of RCI and concomitant therapies, and physicians' assessments of patients who received RCI therapy.

\section{Methods}

This was a retrospective medical record review of a representative sample of patients with uveitis who had been treated with RCI. A nationally representative sample of physicians and patients who used RCI were randomly selected for recruitment from 2 sources, a database of RCI prescribers, and the American Medical Association (AMA) Physician Masterfile. The AMA Physician Masterfile is an up-do-date database of more than 1.4 million physicians, residents, and medical students in the United States. Ophthalmologists from the databases were sent invitations, and all willing physicians with eligible patients were included in the study. Eligible physicians were those who had prescribed RCI to at least 1 patient with uveitis in the past 12 months. Because patients with juvenile rheumatologic conditions such as juvenile rheumatoid arthritis can develop uveitis, physicians who treated pediatric patients (aged 2 years or older) were included. ${ }^{15}$

The study aimed for a total sample of at least 50 cases. Patient inclusion criteria were as follows: (1) a diagnosis of uveitis, (2) received treatment with RCI in the past 12 months,
(3) completed a course of RCI or was receiving RCI treatment at the time of data collection, and (4) complete medical records were available. Patients with infectious eye inflammation, ophthalmic neoplasm, recent ophthalmic or orbital surgery (within 6 months before diagnosis), and recent trauma to the eye (within 60 days before diagnosis) were excluded. These exclusion criteria were intended to exclude patients who did not have noninfectious uveitis. All cases of RCI use were included regardless of contraindications to preserve the observational nature of the study and the physician's decisions about therapy. To ensure that the sample was not dominated by high-volume sites, physicians were permitted to contribute a maximum of 6 cases, which were selected based on the order of last seen as a method of random selection.

Physicians or their designated staff abstracted data from patient medical records using an electronic data collection instrument. The data collection instrument was pretested and refined using actual patient records. It included a physician eligibility screening questionnaire and a patient data collection form. The eligibility screening questionnaire ensured that the physicians had at least 1 eligible patient, had access to the full medical records, and are willing to participate. Abstracted patient data included demographic information, comorbidities, noninfectious uveitis characteristics, treatments for noninfectious uveitis (including RCI and other medications), and physicians' impressions of therapeutic response based on responses to 2 direct questions: "What is the patient's current status?" and "Please select the outcomes that have improved as a result of RCI treatment." Patient characteristics were collected at baseline (before RCI therapy) to characterize the profile of patients who received RCI. Uveitis was classified anatomically according to criteria published by the Standardization of Uveitis Nomenclature Working Group. ${ }^{16}$ Visual impairment was classified based on visual acuity using the World Health Organization (WHO) and International Classification of Diseases, 10th Revision (ICD-10) criteria. $^{17}$

During data collection, physicians had access to technical support. To minimize errors, the data collection software was programmed with real-time and postsurvey data checks. For example, abstractors were notified when an entry was out of range and, where applicable, the data collection tool automatically calculated date ranges, dosing conversions, and other calculated values. Before analysis, the data set was validated and checked for artifacts. To protect confidentiality, all patient and physician data were de-identified at the time of data collection and results are reported in the aggregate. Findings are reported using descriptive statistics. Data were tabulated using IBM SPSS Statistics version 22.0 (IBM Corporation, Armonk, NY).

This article is based on preexisting information and does not contain any direct studies with human participants. Existing de-identified data were used, and no personal health information was collected. An institutional review board (Solutions Institutional Review Board, Little Rock, $\mathrm{AR})$ reviewed the study and verified it as exempt due to the lack of identifiers, use of existing data, and no direct contact with patients [45CFR46.101(b)(4): Existing Data and Specimens-No Identifiers].

\section{Results}

A total of 21 eligible ophthalmologists identified patients and abstracted patient data. Data collection occurred between 
September and October 2017. The final study sample includes 91 patients (Table 1). The mean age was 41 years (standard deviation, 14.1 years; range, $11-78$ years), and $62 \%$ of the patients were female. Patients were primarily Caucasian/nonHispanic (46\%) or African American (32\%), had geographical variations, and had a range of educational backgrounds. The most common comorbidities were hypertension, sarcoidosis, and diabetes, which occurred in $12 \%-18 \%$ of patients. Approximately one-third of patients had no comorbid conditions.

The study patients had been diagnosed with uveitis for a mean of 3.98 years and had experienced a mean of 3.5 acute episodes in the past. The most common anatomic presentation of uveitis was anterior uveitis $(n=38,42 \%)$, followed by intermediate uveitis $(n=19,21 \%)$, posterior uveitis $(n=19,21 \%)$, and diffuse uveitis/panuveitis $(n=15,16 \%)$. Uveitis affected both eyes in 59\% of patients and affected 1 eye in $41 \%$ of patients.

At baseline, uveitis disease severity was mild, moderate, and severe in 5 patients (5\%), 63 patients $(69 \%)$, and 23 patients $(25 \%)$, respectively. Forty-eight patients $(53 \%)$ had moderate visual impairment (visual acuity 20/200 to 20/70), $21(23 \%)$ had severe visual impairment (20/400 to 20/200), and no patients were considered blind according to the WHO and ICD-10 criteria ${ }^{17}$ (Table 2). Signs and symptoms documented at baseline included blurred vision, light sensitivity, floaters, loss of acuity, eye pain, and eye redness. In patients experiencing these signs and symptoms, the severity was most often moderate (Table 3 ).

Patients had used a mean of 2.5 medications (interquartile range, 2-3) before the current RCI regimen. Of 71 patients with documentation of prednisone treatment in their medical record, $50(70 \%)$ had received $10 \mathrm{mg}$ or more per day of oral prednisone therapy for 6 months or longer.

For most patients $(n=83,91 \%)$, RCI treatment in the study period was the patient's first ever use of RCI. The RCI dosing regimens varied among the study patients, with 17 different initial dosing regimens documented. Most patients $(n=70,77 \%)$ were prescribed an initial regimen of 40-80 U of RCI administered subcutaneously once or twice weekly. The course of treatment also varied. At the time of data collection, treatment was ongoing at the initial dose in 27 patients (30\%), with a mean duration of 16.7 weeks. In 27 patients $(30 \%)$, the initial dose was tapered downwards before discontinuation, with a total duration of 7.6 weeks. The reason for discontinuation of RCI is not part of the data collection of the study. In 17 patients (19\%), the initial dose was adjusted downward $(n=10)$ or upward $(n=7)$, with a mean of 12.7 weeks. Twenty patients $(22 \%)$ discontinued RCI after a mean of 4.25 weeks.

Before initiation of RCI, all 91 patients were receiving medications for uveitis. During treatment with RCI, use of these concomitant medications reduced to 52 patients (57\%), and after RCI therapy, the number further reduced to 20 patients $(22 \%)$ (Fig. 1). The concomitant medications included steroid eye drops, oral steroid, intraocular steroid injections, nonsteroid eye drops (e.g., eye drops containing nonsteroidal anti-inflammatory drugs [NSAIDs]), and biologics (e.g., adalimumab). Among patients who remained on concomitant medications, there were dosing reductions. Dosing reductions were observed for 22 of 52 patients using steroid eye drops, 7 of 9 patients using an oral steroid, 1 of 2 patients receiving intraocular steroid injections, 4 of 13
Table 1. Demographic and Clinical Characteristics of Patients With Uveitis Who Were Treated with Repository CORTICOTROPIN INJECTION

\begin{tabular}{|c|c|}
\hline Characteristic & $\begin{array}{c}\text { Patients } \\
\text { with uveitis } \\
(\mathrm{N}=91)\end{array}$ \\
\hline Mean age (SD) in years & $41(14)$ \\
\hline \multicolumn{2}{|l|}{ Age category, $n(\%)$, years } \\
\hline $11-17$ & $3(3)$ \\
\hline $18-24$ & $7(8)$ \\
\hline $25-34$ & $19(21)$ \\
\hline $35-44$ & $26(29)$ \\
\hline $45-54$ & $19(21)$ \\
\hline $55-64$ & $12(13)$ \\
\hline $65+$ & $5(5)$ \\
\hline \multicolumn{2}{|l|}{ Gender, $n(\%)$} \\
\hline Male & $35(38)$ \\
\hline Female & $56(62)$ \\
\hline \multicolumn{2}{|l|}{ Race/ethnicity, $n(\%)$} \\
\hline African American & $29(32)$ \\
\hline American Indian/Alaska Native & $2(2)$ \\
\hline Asian & $5(5)$ \\
\hline Caucasian/Non-Hispanic & $42(46)$ \\
\hline Hispanic/Latino & $8(9)$ \\
\hline Native Hawaiian/Pacific Islander & $1(1)$ \\
\hline Other/not reported & $4(4)$ \\
\hline \multicolumn{2}{|l|}{ Geographic region, $n(\%)$} \\
\hline Northeast & $42(46)$ \\
\hline Midwest & $9(10)$ \\
\hline South & $25(27)$ \\
\hline West & $15(16)$ \\
\hline \multicolumn{2}{|l|}{ Highest level of education completed, $n(\%)$} \\
\hline Associate or bachelor's degree & $27(30)$ \\
\hline $\begin{array}{l}\text { High school graduate, diploma, } \\
\text { or equivalent }\end{array}$ & $24(26)$ \\
\hline Advanced degree & $11(12)$ \\
\hline Nursery school to 8th grade & $3(3)$ \\
\hline Unknown or not reported & $26(29)$ \\
\hline \multicolumn{2}{|l|}{ Comorbidities, $n(\%)$} \\
\hline None & $28(31)$ \\
\hline Hypertension & $16(18)$ \\
\hline Sarcoidosis & $13(14)$ \\
\hline Diabetes & $11(12)$ \\
\hline Ankylosing spondylitis & $8(9)$ \\
\hline Chronic joint disease/rheumatoid arthritis & $7(8)$ \\
\hline Multiple sclerosis & $7(8)$ \\
\hline Hyperlipidemia & $5(5)$ \\
\hline Vision loss & $5(5)$ \\
\hline Gastrointestinal conditions & $3(3)$ \\
\hline Heart conditions & $3(3)$ \\
\hline Metabolic disorder & $3(3)$ \\
\hline Cancer & $2(2)$ \\
\hline Hearing problems & $2(2)$ \\
\hline Juvenile idiopathic arthritis & $2(2)$ \\
\hline Mood disorders & $2(2)$ \\
\hline Polymyositis & $2(2)$ \\
\hline Respiratory conditions & $2(2)$ \\
\hline Urologic and kidney disorders & $2(2)$ \\
\hline Gout & $1(1)$ \\
\hline Other CNS conditions & $1(1)$ \\
\hline
\end{tabular}

CNS, central nervous system; SD, standard deviation. 
Table 2. Level of Visual Impairment at Baseline in Patients with Uveitis Who Were Treated WITH REPOSITORY CORTICOTROPIN INJECTION

\begin{tabular}{lcc}
\hline Visual impairment, $\mathrm{n}(\%)$ & One eye $(\mathrm{n}=37)$ & Both eyes $(\mathrm{n}=54)$ \\
\hline Mild or none (better than 20/70) & $10(27)$ & $11(20)$ \\
Moderate (worse than 20/70, better than 20/200) & $18(49)$ & $30(56)$ \\
Severe (worse than 20/200, better than 20/400) & $9(24)$ & $12(22)$ \\
Blindness, level 3 (worse than 20/400, better than 5/300) & 0 & 0 \\
Blindness, level 4 (worse than 5/300) & 0 & 0 \\
Blindness, level 5 (no light perception) & 0 & $1(2)$ \\
Undetermined/unspecified & 0 & 0 \\
\hline
\end{tabular}

patients using nonsteroid eye drops, and 2 of 4 patients receiving biologics.

In response to the question "What is the patient's current status?" physicians categorized 76 patients $(84 \%)$ as "improved" after RCI treatment, 15 patients $(16 \%)$ as the "same," and no patients as "worsened" (Table 4). In addition, physicians were asked to select the outcomes that have improved as a result of RCI treatment. Improvements were reported across numerous clinical outcomes; improvements in vision were reported in most patients $(n=78$, $86 \%)$, followed by improvements in pain $(n=25,27 \%)$, and improvements in vitreous haze $(n=24,26 \%)$ (Table 4).

Improved clinical status did not appear to be associated with any particular RCI utilization pattern. An "improved" status was seen in $89 \%$ of patients (24/27) who continued their initial doses of RCI, $81 \%$ of patients (22/27) whose doses were tapered from their initial doses, $82 \%$ of patients (14/17) whose doses were adjusted, and 85\% of patients (17/ 20) who discontinued RCI during the study period.

\section{Discussion}

This study reports medication utilization patterns and physician's assessments of disease status in 91 patients with uveitis who received treatment with RCI. Patients had been diagnosed with uveitis for an average of 4 years and had used $2-3$ other prior medications, often including $10 \mathrm{mg} /$ day or higher doses of corticosteroids for 6 months or longer. The utilization patterns observed indicate that physicians use an individualized approach to RCI regimens. Many patients discontinued or reduced their dose of concomitant uveitis medications during and after RCI therapy. This reduction in concomitant uveitis medication use was noted

Table 3. Uveitis Symptoms and Severity Before INITIATION OF REPOSITORY CORTICOTROPIN INJECTION

\begin{tabular}{|c|c|c|c|c|}
\hline \multirow{2}{*}{$\begin{array}{l}\text { Sign and } \\
\text { symptom }^{\mathrm{a}}\end{array}$} & \multirow{2}{*}{$\begin{array}{l}\text { Sign and } \\
\text { symptom } \\
\text { present? } \\
(\mathrm{N}=91) \\
\mathrm{n}(\%)\end{array}$} & \multicolumn{3}{|c|}{ Severity (No. of patients) } \\
\hline & & Mild & Moderate & Severe \\
\hline Blurred vision & $81(89)$ & 10 & 52 & 19 \\
\hline Light sensitivity & $41(45)$ & 9 & 22 & 10 \\
\hline Floaters & $40(44)$ & 12 & 25 & 3 \\
\hline Visual loss/acuity & $40(44)$ & 5 & 24 & 11 \\
\hline Eye pain & $34(37)$ & 9 & 20 & 5 \\
\hline Eye redness & $30(33)$ & 6 & 22 & 2 \\
\hline
\end{tabular}

${ }^{\mathrm{a}}$ Respondents could select all options that applied; sum exceeds $100 \%$. across all medication classes, including oral steroids, intraocular and topical ophthalmic steroids, nonsteroidal topical ophthalmic agents, and biologics. After RCI therapy, physicians reported improvement for most patients (84\%) in response to the question "what is the patient's current status?"; physicians further reported improvements in vision ( $86 \%$ of patients), pain (27\% of patients), and vitreous haze (26\% of patients) in response to the data collection item "Please select the outcomes that have improved as a result of RCI treatment."

Because the use of RCI is not widespread for uveitis, it presents a challenge to perform real-world studies of utilization and outcomes in this patient population. To our knowledge, this is the largest case series report to date describing patients with uveitis treated with RCI. The published literature on this topic is limited to 2 case reports. Previously, Agarwal et al. described the case of a single adult male with recurrent uveitis who had previously responded to tocilizumab therapy, but was denied coverage for a new episode. ${ }^{13}$ This patient received $80 \mathrm{U}$ of RCI administered subcutaneously 2 times per week for the recurrence, and he experienced a reduction in retinal vascular leakage, improvements in macular edema and optic nerve inflammation, and resolution of vitritis after 6 weeks of RCI therapy. This patient maintained 20/20 visual acuity and did not report any systemic or local adverse effects. In a second case report, a 16-year-old female with recurrent uveitis associated with poorly controlled juvenile idiopathic arthritis reported remission of uveitis symptoms after receiving RCI ( $80 \mathrm{U}$ administered intramuscularly 2 times per week) as additive therapy. ${ }^{14}$ Notably, before RCI therapy, she reported 1 to 2 uveitis flares per month despite ongoing systemic therapy, including steroids, methotrexate, and infliximab. Remission of ocular symptoms was achieved within 2 weeks of initiating RCI and continued for at least 6 months. It is important to note that these case reports may not be representative of outcomes in the overall patient population.

Physicians in the current study used individualized dosing strategies to reach therapeutic goals. When initial dosage, treatment duration, and dose adjustments are taken into account, no 2 patients in the study received the same RCI regimen. The Ocular Immunology and Uveitis Foundation (OIUF) recommends that durable remission is the primary goal of treatment, using any approach that is necessary to achieve that goal while also minimizing the potential for harm or impact on quality of life. ${ }^{18}$ The finding of this study shows that similar proportions of patients across different RCI regimens had achieved improved status, suggesting that physicians are tailoring RCI dosing regimens based on each 


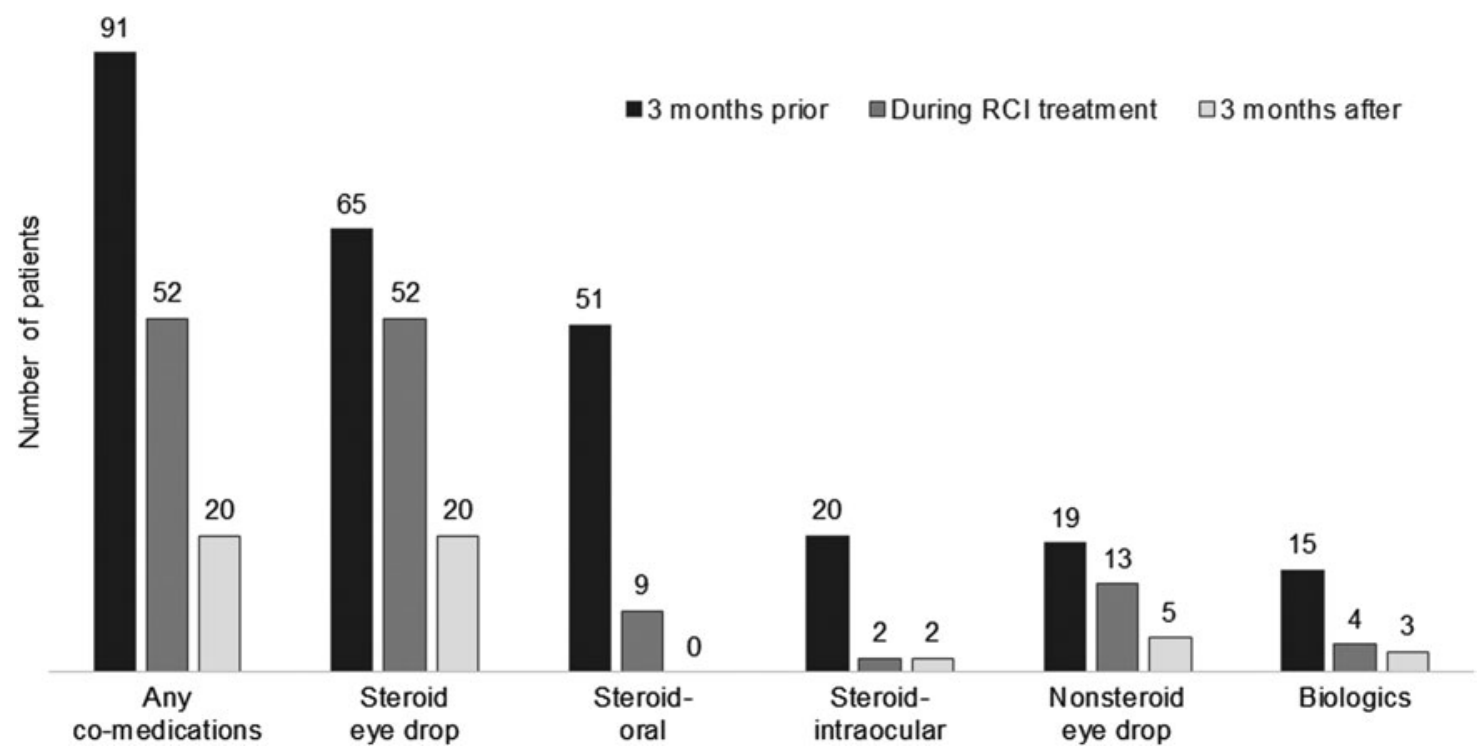

FIG. 1. Concomitant medication use in patients with uveitis 3 months before, during, and 3 months after treatment with RCI $(N=91)$. RCI, repository corticotropin injection.

patient's specific case, an approach that accords with the recommendations of OIUF.

As noted above, this study's findings suggest a potential for decreasing background medication use during and after RCI therapy for noninfectious uveitis. These findings are hypothesis-generating and require additional studies for further evaluation. Decreased use of corticosteroids, in particular, is an important treatment objective. Corticosteroids are not appropriate for long-term use in patients with uveitis, because they increase the risks of developing cataract and glaucoma that could further worsen vision. ${ }^{18,19}$ While corticosteroids are effective and typically used as firstline therapy to rapidly control acute inflammation, they are associated with significant adverse effects, including skin and

Table 4. Physicians' Assessments of Patients WITH UVEITIS Following REPOSITORY CORTICOTROPIN INJECTION TREATMENT

\begin{tabular}{lc}
\hline Category & $\mathrm{N}(\%), \mathrm{N}=91$ \\
\hline Patient's current status $^{\mathrm{a}}$ & $76(84)$ \\
$\quad$ Improved & $15(16)$ \\
Same & 0 \\
$\quad$ Worsened & $78(86)$ \\
Type of improvement & \\
Improvements in vision $_{\text {Improvements in pain }}$ & $25(27)$ \\
Improvements in vitreous haze & $24(26)$ \\
Reduction of background medication use & $22(24)$ \\
Improvements in vitreous flare & $21(23)$ \\
Improvements in macular edema & $16(18)$ \\
\hline
\end{tabular}

'Physicians' response to the question "What is the patient's current status?",

'Physicians' response to the question "Please select the outcomes below that have improved as a result of RCI treatment." Available options included all those shown in the table. Respondents may select all options that apply; sum exceed $100 \%$.

${ }^{\mathrm{c}}$ As measured by optical coherence tomography.

RCI, repository corticotropin injection. subcutaneous tissue disorders, laboratory abnormalities (e.g., hyperglycemia, dyslipidemia), ${ }^{20}$ weight changes, infections, musculoskeletal and connective tissue disorders, and psychiatric disorders. ${ }^{21}$ The adverse effects of RCI are related primarily to its steroidogenic effects. Specifically, RCI stimulates low levels of cortisol release ${ }^{22}$; In addition, RCI is believed to work via a different pathway by binding to specific melanocortin receptors. ${ }^{12}$ In an analysis of data from 2 clinical trials, corticosteroid-related adverse events in patients with uveitis were found to be frequent and dose-dependent, with increasing corticosteroid dose associated with higher incidence of adverse effects. The analysis reported an incidence of 454 and 317 per 100 patient-years before dose tapering in the placebo groups of the VISUAL-1 (Efficacy and Safety of Adalimumab in Patients with Active Uveitis) and VISUAL-2 (Efficacy and Safety of Adalimumab in Subjects with Inactive Uveitis) clinical trials, respectively. ${ }^{21}$

This study has several limitations. We relied on retrospective collection of data from medical records, which may be incomplete. However, our data collection focused on the clinical aspects of each case, based on data available in the medical record. The study's aim is to describe the characteristics of patients who were prescribed RCI; therefore, the study scope did not include quantification of the patients' outcomes, such as diagnostic measurements or safety endpoints. Another limitation is that physician assessment of a patient's improvement following therapy may be subjective, and physicians may have varying definitions of improvement. Although physicians may have used objective measures, such as diagnostic tests, to inform their assessments, we did not collect those data. Finally, as an observational study, the physicians' assessments reported in this study might be related to therapies not documented in the medical records.

This descriptive study has identified several areas of future research. A detailed quantification of concomitant medications and reduction in their use as well as identification of the reasons behind the treatment patterns observed herein would further advance our knowledge. Studies to 
quantify the clinical response and evaluate real-world safety of RCI in patients with uveitis would also be valuable.

This study did not capture information on adverse reactions in this group of patients with uveitis, who were receiving RCI and a variety of other concomitant medications, including corticosteroids. Published safety results of RCI in patients with uveitis are limited to 2 cases, both of whom were also receiving other concomitant therapies, including corticosteroids. $^{13,14}$ There were no adverse events reported in either patient after 6 weeks and 6 months of RCI treatment, respectively, including no local ocular or dermal injection site events. Potential common adverse reactions to RCI include fluid retention, alteration in glucose tolerance, elevation in blood pressure, behavioral and mood changes, increased appetite, and weight gain. ${ }^{12}$ These side effects are similar to those known to occur with the corticosteroid class of uveitis treatments. ${ }^{4}$

The study sample is nationally representative of physicians and patients who use RCI because it is a national probability sample. Our study obtained, from 2 national sources, a random sample of physicians who manage studyeligible patients, and then further obtained a random sample of the patients under their care. Thus, the findings may be generalizable to the broader population of US patients with uveitis who received RCI therapy.

In summary, we found that RCI was used in patients with uveitis who had, on average, a 4-year history of the condition, extensive prior corticosteroid treatment, and prior treatment with 2 or 3 other therapies. Dosing regimens were individualized, suggesting that physicians are treating to a target therapeutic goal that may differ for each patient. Use of other uveitis medications decreased after initiation of RCI therapy. Physicians reported improvements in the patients' status following RCI therapy for $84 \%$ of the study patients. Improvements in vision were reported for $86 \%$, followed by improvements in pain (27\%), and improvements in vitreous haze $(26 \%)$. The study findings support the use of RCI as a treatment option for uveitis and provide a better understanding of patient characteristics and practice patterns that may contribute to its appropriate use.

\section{Acknowledgments}

Additional editorial assistance: Denise Galipeau of Global Outcomes Group provided medical writing services, and Esther Tazartes and Judith Hurley of Global Outcomes Group provided editorial assistance; these services were funded by Mallinckrodt Pharmaceuticals (Hazelwood, MO). Sponsorship for this study and article processing charges were funded by Mallinckrodt Pharmaceuticals.

\section{Author Disclosure Statement}

All authors had full access to all of the data in this study. All named authors meet the International Committee of Medical Journal Editors (ICJME) criteria for authorship for this article, take responsibility for the integrity of the work as a whole, and have given approval for this version to be published. W.W.N., A.F.L., J.K., B.O.-O., and G.C. are employees of Mallinckrodt Pharmaceuticals and declare that they have no other conflicts of interest. J.R.G., K.H., and S.C. were research consultants for the study and declare that they have no conflicts of interest.

\section{References}

1. Barisani-Asenbauer, T., Maca, S.M., Mejdoubi, L., Emminger, W., Machold, K., and Auer, H. Uveitis-a rare disease often associated with systemic diseases and infections-a systematic review of 2619 patients. Orphanet. J. Rare Dis. 7: 57, 2012.

2. Mustafa, M., Muthusamy, P., Hussain, S.S., Shimmi, S.C., and Sein, M.M. Uveitis: pathogenesis, clinical presentations and treatment. IOSR J. Pharm. 4:42-47, 2014.

3. Guly, C.M., and Forrester, J.V. Investigation and management of uveitis. BMJ 341:c4976, 2010.

4. Barry, R.J., Nguyen, Q.D., Lee, R.W., Murray, P.I., and Denniston, A.K. Pharmacotherapy for uveitis: current management and emerging therapy. Clin. Ophthalmol. 8: 1891-1911, 2014.

5. Acharya, N.R., Tham, V.M., Esterberg, E., Borkar, D.S., Parker, J.V., Vinoya, A.C., and Uchida, A. Incidence and prevalence of uveitis: results from the Pacific Ocular Inflammation Study. JAMA Ophthalmol. 131:1405-1412, 2013.

6. Gritz, D.C., and Wong, I.G. Incidence and prevalence of uveitis in Northern California; the Northern California Epidemiology of Uveitis Study. Ophthalmology 111:491500; discussion 500, 2004.

7. Suhler, E.B., Lloyd, M.J., Choi, D., Rosenbaum, J.T., and Austin, D.F. Incidence and prevalence of uveitis in Veterans Affairs Medical Centers of the Pacific Northwest. Am. J. Ophthalmol. 146:890.e8-896.e8, 2008.

8. Darrell, R.W., Wagener, H.P., and Kurland, L.T. Epidemiology of uveitis. Incidence and prevalence in a small urban community. Arch. Ophthalmol. 68:502-514, 1962.

9. Durrani, O.M., Tehrani, N.N., Marr, J.E., Moradi, P., Stavrou, P., and Murray, P.I. Degree, duration, and causes of visual loss in uveitis. Br. J. Ophthalmol. 88:1159-1162, 2004.

10. Albini, T., Rice, B., White, A., Lopez, A., Reiff, J., Lima, F., Filas, B.A., Elliott, S.B., Bartels-Peculis, L., Ciepielewska, G., and Nelson, W.W. Clinical outcomes, healthcare resource use, and cost in patients with noninfections inflammatory eye disease (NIIED) in a US population. In: American Academy of Ophthalmology (AAO) Annual Meeting, New Orleans, LA; 2017.

11. Dick, A.D., Tundia, N., Sorg, R., Zhao, C., Chao, J., Joshi, A., and Skup, M. Risk of ocular complications in patients with noninfectious intermediate uveitis, posterior uveitis, or panuveitis. Ophthalmology 123:655-662, 2016.

12. H.P. Acthar Gel (repository corticotropin injection) [prescribing information]. Hazelwood, MO: Mallinckrodt Pharmaceuticals; 2017.

13. Agarwal, A., Hassan, M., Sepah, Y.J., Do, D.V., and Nguyen, Q.D. Subcutaneous repository corticotropin gel for non-infectious panuveitis: reappraisal of an old pharmacologic agent. Am. J. Ophthalmol. Case Rep. 4:78-82, 2016.

14. Ahmad, K.T., Sukumaran, S., Brown, K.L., and Ali, T.K. Use of corticotropin hormone for treatment of refractory uveitis secondary to juvenile idiopathic arthritis. In 9th International Symposium on Uveitis, Dublin, Ireland; August 18-21, 2016.

15. Gregory, A.C., 2nd, Kempen, J.H., Daniel, E., Kacmaz, R.O., Foster, C.S., Jabs, D.A., Levy-Clarke, G.A., Nussenblatt, R.B., Rosenbaum, J.T., Suhler, E.B., and Thorne, J.E.; Systemic Immunosuppressive Therapy for Eye Diseases Cohort Study Research Group. Risk factors for loss of visual acuity among patients with uveitis associated with juvenile idiopathic arthritis: the Systemic Immunosuppressive Therapy for Eye Diseases Study. Ophthalmology 120:186-192, 2013. 
16. Jabs, D.A., Nussenblatt, R.B., and Rosenbaum, J.T.; Standardization of Uveitis Nomenclature (SUN) Working Group. Standardization of uveitis nomenclature for reporting clinical data. Results of the First International Workshop. Am. J. Ophthalmol. 140:509-516, 2005.

17. Dandona, L., and Dandona, R. Revision of visual impairment definitions in the International Statistical Classification of Diseases. BMC Med. 4:7, 2006.

18. Foster, C.S., Kothari, S., Anesi, S.D., Vitale, A.T., Chu, D., Metzinger, J.L., and Ceron, O. The Ocular Immunology and Uveitis Foundation preferred practice patterns of uveitis management. Surv. Ophthalmol. 61:1-17, 2016.

19. American Academy of Ophthalmology. Practicing ophthalmologists curriculum 2014-2016: uveitis. San Francisco, CA: American Academy of Ophthalmology; 2014.

20. Liu, D., Ahmet, A., Ward, L., Krishnamoorthy, P., Mandelcorn, E.D., Leigh, R., Brown, J.P., Cohen, A., and Kim, $\mathrm{H}$. A practical guide to the monitoring and management of the complications of systemic corticosteroid therapy. Allergy Asthma Clin. Immunol. 9:30, 2013.

21. Suhler, E.B., Thorne, J.E., Mittal, M., Betts, K.A., Tari, S., Camez, A., Bao, Y., and Joshi, A. Corticosteroid-related adverse events systematically increase with corticosteroid dose in noninfectious intermediate, posterior, or panuveitis: post hoc analyses from the VISUAL-1 and VISUAL-2 trials. Ophthalmology 124:1799-1807, 2017.

22. Lal, R., Bell, S., Challenger, R., Hammock, V., Nyberg, M., Decker, D., Becker, P.M., and Young, D. Pharmacodynamics and tolerability of repository corticotropin injection in healthy human subjects: a comparison with intravenous methylprednisolone. J. Clin. Pharmacol. 56:195-202, 2016.

Received: July 26, 2018 Accepted: December 13, 2018

Address correspondence to: Dr. Winnie W. Nelson Mallinckrodt Pharmaceuticals 1425 U.S. Route 206 Bedminster, NJ 07921

E-mail: winnie.nelson@mnk.com; tom@globaloutcomesgroup.com 